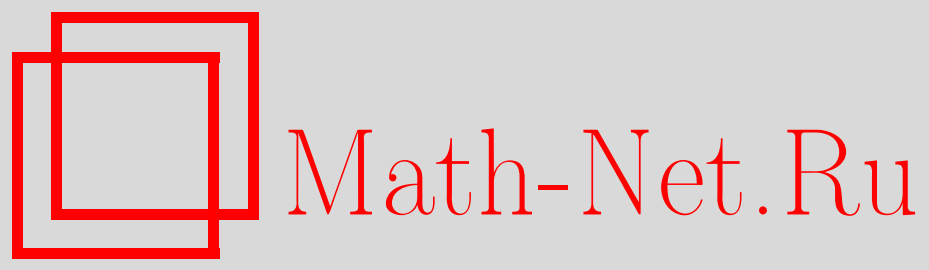

А. В. Карабегов, Конструкция канонической следовой плотности деформационного квантования с разделением переменных, Функи. анализ и его прил., 1998, том 32, выпуск 4, 85-87

DOI: https://doi.org/10.4213/faa440

Использование Общероссийского математического портала MathNet.Ru подразумевает, что вы прочитали и согласны с пользовательским соглашением http://www.mathnet.ru/rus/agreement

Параметры загрузки:

IP : 54.196 .121 .252

26 апреля 2023 г., 07:49:30

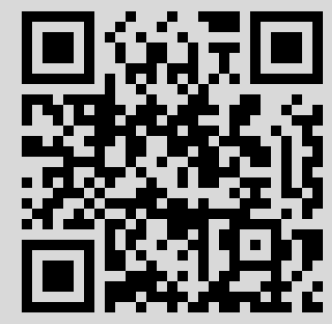


Автор благодарен рецензенту за ценные замечания.

\title{
ЛИТЕРАТУРА
}

1. Nahm W. Quantum field theories in one and two dimensions. Duke Math. J., 54, No. 2, 579-613 (1987).

Московский государственный

технический университет им. Н. Э. Баумана

Поступило в редакцию

11 июня 1997 г.

УДК 517.9

\section{Конструкция канонической следовой плотности деформационного квантования с разделением переменных}

\author{
(C) 1998. А. В. КАРАБЕГОВ
}

В данной заметке мы приводим явную конструкцию канонической следовой плотности (см. [1]) деформационного квантования с разделением переменных на псевдокэлеровом многообразии, введенного в [2].

1. Для данного векторного пространства $V$ назовем формальными векторами элементы пространства $\left.V\left[\nu^{-1}, \nu\right]\right]=\left\{v=\sum_{j \geqslant k} \nu^{j} v_{j} \mid v_{j} \in V, k \in \mathbb{Z}\right\}$ формальных рядов Лорана от параметра $\nu$. Так мы определяем поле формальных чисел $\left.\mathscr{K}=\mathbb{C}\left[\nu^{-1}, \nu\right]\right]$, формальные функции, формы и т. д.

Пусть $M$ есть $2 n$-мерное симплектическое многообразие с симплектической формой $\omega_{-1}$ и соответствующей скобкой Пуассона $\{\cdot, \cdot\}, U-$ произвольное открытое подмножество в $M$ и $\left.\mathscr{F}(U)=C^{\infty}(U)\left[\nu^{-1}, \nu\right]\right], \mathscr{F}_{0}(U)=$ $\left.C_{0}^{\infty}(U)\left[\nu^{-1}, \nu\right]\right]$ - пространства формальных функций на $U$.

Пусть на $\mathscr{F}(M)$ определено ассоциативное, непрерывное в $\nu$-адической топологии $\mathscr{K}$-линейное умножение $\star$ ( $\star$-умножение $)$, заданное на функциях $f, g \in C^{\infty}(M)$ формулой $f \star g=\sum_{r \geqslant 0} \nu^{r} C_{r}(f, g)$, где $C_{r}, r \geqslant 0$, суть билинейные дифференциальные по обоим аргументам операторы, такие, что $C_{0}(f, g)=f g$ и $C_{1}(f, g)-C_{1}(g, f)=i\{f, g\}$. Тогда говорят, что на $M$ задано деформационное квантование (см. [3]).

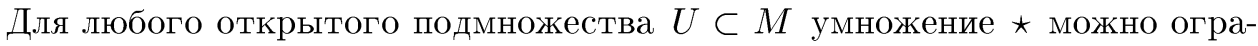
ничить на $U$. Умножение на $\mathscr{F}(U)$ будем обозначать по-прежнему через $\star$.

Следовой плотностью умножения $\star$ на $U$ называется гладкая формальная плотность $d \mu$ на $U$, такая, что для любых $f, g \in \mathscr{F}_{0}(U)$ выполняется соотношение $\int_{U} f \star g d \mu=\int_{U} g \star f d \mu$. Б. В. Федосов построил в [1] глобальную следовую плотность $d \mu_{\text {can }}$ умножения $\star$ на симплектическом многообразии $\left(M, \omega_{-1}\right)$. Эта плотность, называемая канонической, играет важную роль в теоремах об индексе в деформационном квантовании. Старший член формального ряда $d \mu_{\text {саn }}$ равен $(2 \pi \nu)^{-n} \omega_{-1}^{n} / n !$.

Любая следовая плотность $d \mu$ на $U \subset M$ представима в виде $d \mu=\lambda d \mu_{\text {can }}$, где $\lambda$ - локально постоянная формальная функция на $U$. 
Известно, что на достаточно малой окрестности $U$ любой точки многообразия $M$ существует дифференцирование $\delta$ алгебры $(\mathscr{F}(U), \star)$ вида $\delta=\partial / \partial \nu+A$, где $A$ - формальный дифференциальный оператор на $U$. Можно показать, что для любой формальной функции $f \in \mathscr{F}_{0}(U)$ выполняется соотношение $(d / d \nu) \int_{U} f d \mu_{\text {can }}=\int_{U} \delta(f) d \mu_{\text {can }}$.

Предположим теперь, что $U$ связна и на ней задана ненулевая следовая плотность $d \mu=\lambda d \mu_{\text {сап }}$ умножения $\star$. Тогда $\lambda$ - ненулевая формальная константа, $\lambda \in \mathscr{K} \backslash\{0\}$, и для $f \in \mathscr{F}_{0}(U)$ выполняется соотношение $(d / d \nu) \int_{U} f d \mu$ $-\int_{U} \delta(f) d \mu=(d \lambda / d \nu) \lambda^{-1} \int_{U} f d \mu$, откуда мы можем определить элемент $\lambda$ с точностью до постоянного множителя. Этот множитель можно вычислить, приравнивая старшие члены формальных рядов $d \mu$ и $\lambda d \mu_{\text {can }}$. Таким образом по $d \mu$ можно восстановить каноническую следовую плотность умножения $\star$ на $U, d \mu_{\text {can }}=\lambda^{-1} d \mu$.

2. Пусть на $M$ задано $\star$-умножение . Для любого открытого подмножества $U \subset M$ и $f, g \in \mathscr{F}(U)$ обозначим через $L_{f}$ оператор левого $\star$ умно-

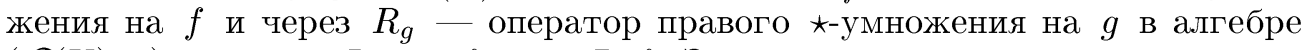
$(\mathscr{F}(U), \star)$, так что $L_{f} g=f \star g=R_{g} f$. Эти операторы формальные дифференциальные. Обозначим через $\mathscr{L}(U)$ и $\mathscr{R}(U)$ множества всех операторов левого и правого ^-умножения на $U$ соответственно.

Пусть теперь на $M$ задана комплексная структура, для которой симплектическая форма $\omega_{-1}$ имеет тип $(1,1)$, т.е. является псевдокэлеровой формой. Тогда $\left(M, \omega_{-1}\right)$ - псевдокэлерово многообразие. Деформационное квантование на $\left(M, \omega_{-1}\right)$ называется квантованием с разделением переменных, если на любом открытом подмножестве $U \subset M \star$-умножение на голоморфную функцию $a$ слева и $\star$-умножение на антиголоморфную функцию $b$ справа совпадают с поточечным умножением на эти функции, $L_{a}=a$, $R_{b}=b$.

Деформационные квантования, полученные из виковского и антивиковского квантований на $\mathbb{C}^{n}$ и из квантования Березина в [4-7] асимптотическим разложением произведения символов по $\hbar \rightarrow 0$, являются квантованиями с разделением переменных (здесь имеется в виду «постоянная Планка» $\hbar$, входящая в определение квантования Березина).

В работе [2] показано, что деформационные квантования с разделением переменных находятся во взаимно однозначном соответствии с формальными деформациями псевдокэлеровой формы $(1 / \nu) \omega_{-1}$, т. е. с формальными рядами $\omega=\sum_{r \geqslant-1} \nu^{r} \omega_{r}$, где $\omega_{r}, r \geqslant 0$, - замкнутые $(1,1)$-формы. А именно, пусть на произвольной стягиваемой координатной окрестности $U \subset M$ с голоморфными координатами $\left\{z^{1}, \ldots, z^{n}\right\}$ задан формальный потенциал $\Phi$ формы $\omega$, т.е. $\omega=i \partial \bar{\partial} \Phi$. Множество операторов левого $\star$-умножения $\mathscr{L}(U)$ состоит из формальных дифференциальных операторов на $U$, коммутирующих с операторами умножения на антиголоморфные функции и с операторами $\partial \Phi / \partial \bar{z}^{l}+\partial / \partial \bar{z}^{l}$. Зная $\mathscr{L}(U)$, легко восстановить -умножение на $U$ по формуле $f \star g=L_{f} g, f, g \in \mathscr{F}(U)$.

Для данного деформационного квантования с разделением переменных на псевдокэлеровом многообразии $\left(M, \omega_{-1}\right)$ со $\star$-умножением $\star$ существует единственный формальный дифференциальный оператор $I$ на $M$, такой, что для любого открытого подмножества $U \subset M$, любой голоморфной функции 
$a$ и антиголоморфной функции $b$ на $U$ выполняется соотношение $I(a b)=$ $b \star a$ (здесь имеется в виду ограничение $I$ на $U)$. Легко проверить, что $I=1+\nu \Delta+\ldots$, где $\Delta$ - оператор Лапласа-Бельтрами, отвечающий псевдокэлеровой метрике на $\left(M, \omega_{-1}\right)$. В частности, $I$ обратим. Можно показать, что для деформационных квантований, полученных из виковского квантования на $\mathbb{C}^{n}$ и из квантования Березина на орбитах компактной полупростой группы Ли, оператор $I$ получается из асимптотического разложения по $\hbar \rightarrow 0$ преобразования Березина, отображающего контравариантные символы в соответствующие ковариантные (см. [8]). Естественно назвать $I$ формальным преобразованием Березина. Введем новое умножение ґ на $\mathscr{F}(M)$ формулой $f \tilde{\star} g=I^{-1}(I g \star I f), f, g \in \mathscr{F}(M)$.

ЛЕмма 1. Умножение $\tilde{\star}$ на $\mathscr{F}(M)$ определяет деформаиионное квантование $c$ разделением переменных на псевдокэлеровом многообразии $\left(M,-\omega_{-1}\right)$.

Обозначим через $\widetilde{\omega}$ формальную деформацию псевдокэлеровой формы

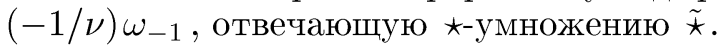

ТЕОРема 1. На любой стягиваемой координатной окрестности $U \subset M$ с голоморфньлми координатами $\left\{z^{1}, \ldots, z^{n}\right\}$, на которой задан формальньий

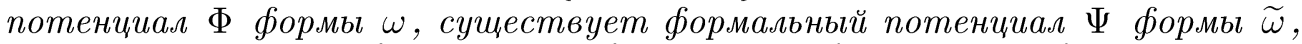
такой, что $I\left(\partial \Psi / \partial z^{k}\right)=-\partial \Phi / \partial z^{k}$ u $I\left(\partial \Psi / \partial \bar{z}^{l}\right)=-\partial \Phi / \partial \bar{z}^{l}$. Плотность $d \mu=\exp (\Phi+\Psi) d z^{1} \cdots d z^{n} d \bar{z}^{1} \cdots d \bar{z}^{n}$ является следовой плотностью умножения на $U$.

ТЕОРема 2 (см. [9]). На произвольном стягиваемом открытом подмножестве $U \subset M$, на котором задан формальный потенииал $\Phi$ формь $\omega$, оператор $\delta=d / d \nu+d \Phi / d \nu-R_{d \Phi / d \nu}$ является дифферениированием в алгебpe $(\mathscr{F}(U), \star)$.

В обозначениях теоремы 1 выразим на окрестности $U$ каноническую сле-

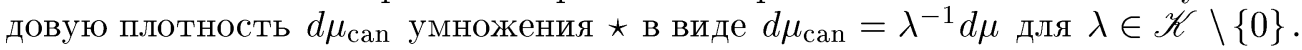
Пользуясь теоремами 1 и 2 , можно показать, что $\lambda$ определяется с точностью до постоянного множителя из уравнения $(d \lambda / d \nu) \lambda^{-1}=I(d \Psi / d \nu)+d \Phi / d \nu$. Этот множитель вычисляется, как в п. 1.

Автор хотел бы выразить свою глубокую благодарность Б. В. Федосову за стимулирующие беседы и рецензентам за ценные замечания.

\section{ЛИТЕРАТУРА}

1. Fedosov B. V. J. Diff. Geom., 40, 213-238 (1994). 2. Karabegov A. V. Comm. Math. Phys., 180, 745-755 (1996). 3. Bayen F., Flato M., Fronsdal C., Lichnerovich A., Sternheimer D. Ann. Phys., 111, 61-151 (1978). 4. Moreno C. Lett. Math. Phys., 11, 361-372 (1986). 5. Moreno C. Lett. Math. Phys., 12, 217-229 (1986). 6. Cahen M., Gutt S., Rawnsley J. Trans. Amer. Math. Soc., 337, 73-98 (1993). 7. Cahen M., Gutt S., Rawnsley J. Lett. Math. Phys., 34, 159-168 (1995). 8. Березин Ф. А. ДАН СССР, 241, № 1, 15-17 (1978). 9. Karabegov A. V. Lett. Math. Phys., 43, 347-357 (1998). 